\title{
25 Research Square \\ Ginkgolide B derivative synthesis and their effects on the viability of SKOV3 cells
}

\section{Zi-Li Feng}

Shaanxi University of Technology

\section{Zhi-Bin Zhu}

Shaanxi University of Technology

Wang Chen ( $\square$ chenwang@snut.edu.cn )

Shaanxi University of Technology https://orcid.org/0000-0003-0565-9157

\section{Jia-Yuan Xu}

Shaanxi University of Technology

\section{Yu Bai}

Shaanxi University of Technology

\section{Dai-Hua Hu}

Shaanxi University of Technology

\section{Zheng-Dong Zhao}

Shaanxi Cuicheng Biomedical Tehnology CO., Ltd.

\section{Research Article}

Keywords: ginkgolide, derivatives, ovarian cancer, SKOV3 cell viability, apoptosis

Posted Date: March 10th, 2021

DOI: https://doi.org/10.21203/rs.3.rs-266392/v1

License: (c) (1) This work is licensed under a Creative Commons Attribution 4.0 International License.

Read Full License 


\section{Abstract}

The natural product GB was used as a raw material and modified by esterification on $\mathrm{C} 10-\mathrm{OH}$ or $\mathrm{C} 1-\mathrm{OH}$ to obtain 11 derivatives (1 to 11), which were structurally characterized with nuclear magnetic resonance spectroscopy. An MTT assay-based in vitro tumor proliferation inhibitory activity test showed that compounds $2,3,6,7,10$, and 11 exhibited strong inhibitory activity against the human ovarian cancer cells SKOV3, with $\mathrm{IC}_{50}$ values of $16.05 \mu \mathrm{mol} / \mathrm{L}, 15.65 \mu \mathrm{mol} / \mathrm{L}, 32.00 \mu \mathrm{mol} / \mathrm{L}, 63.30 \mu \mathrm{mol} / \mathrm{L}, 23.20 \mu \mathrm{mol} / \mathrm{L}$, and $31.10 \mu \mathrm{mol} / \mathrm{L}$, respectively. Annexin V/PI double staining assay showed that compound 2 induced apoptosis in SKOV3 cells to a slightly greater extent than GB and compounds $\mathbf{5}$, and $\mathbf{9}$, with an apoptosis rate of $31.68 \%$.

\section{Introduction}

Ginkgolide B (GB) is a diterpenoid isolated from Ginkgo biloba, which is associated with various pharmacological activities, such as anti-platelet aggregation, anti-inflammatory, antioxidant, and free radical scavenging [1-5]. In recent years, GB has also been reported to have anti-tumor effects [6]; it inhibits the growth and proliferation of various tumor cells, such as ovarian, breast, lung, colorectal, bladder, and prostate cancers, with a rich set of anti-tumor targets and pathways [7-15].

The PAF/PAFR signaling axis has emerged as an important determinant of the aggressive phenotype in several malignancies, including ovarian cancer [16-17]. GB, as a potent PAFR antagonist, may be a key regulator in treating tumor cells. Jiang Wei et al. [7-8] conducted in vivo and in vitro experiments and found that GB could inhibit the proliferation of ovarian cancer cells and growth of nude mice tumors with a tumor suppression rate of $48 \%$, and up to $78.2 \%$ in combination with cisplatin (CDDP), which could be used as an adjuvant drug for the treatment of ovarian cancer. The mechanism may be that GB inhibits the expression of platelet-activating factor (PAF), platelet-activating factor receptor (PAFR), and the tyrosine kinase Src and the p38 mitogen-activated protein kinase (p38MAPK), preventing p38MAPK from activating downstream transcription factors [18], including the cyclic adenosine monophosphate (cAMP) response element binding protein (CREB), which is closely related to cell invasion. This results in the inability of CREB to bind to the promoter in the upstream of the matrix metalloproteinase 2/9 (MMP2/MMP9) gene and the inability of MMP2 mRNA/MMP9 mMRA to be expressed, which inhibits the migration of ovarian cancer cells. In addition, ginkgolide B upregulated 25 proteins with antitumor effects (e.g., p53) and downregulated 22 proteins associated with tumor migration (e.g., $\beta$-catenin).

Yu Y et al. [19] demonstrated that the PAFR upregulation in CDDP-treated ovarian cancer cells provided additional support for the function of the PAF/PAFR signaling axis as a resistance mechanism, with the possible mechanism being that cisplatin (CDDP) acts on the ovarian cancer cells and causes nuclear factor kappa-B (NF-KB) and hypoxia-inducible factor (HIF-1 a) to accumulate in the nucleus, leading to the upregulation of the platelet-activating factor receptor PAFR. In addition, GB inhibited the PAFR activity, which may block the downstream signaling pathways of phosphoinositide 3-kinase (PI3K) and the 
extracellular regulated protein kinase (ERK), thereby enhancing the sensitivity of ovarian cancer cells to CDDP, intensifying drug efficacy and significantly reducing tumor growth.

Ginkgolide $B$ has a unique rigid caged dodeca-carbon skeleton structure, and it was found that the synthesis of GB ester derivatives by converting the hydroxyl groups at the C-1 and (or) C-10 positions of GB into aromatic-containing groups such as ester groups significantly enhanced the anti-PAF activity [20-21]. To explore the effect of GB parent structure and the introduction of small molecule side chains of benzoic acid series on the hydroxyl groups at C-1 and (or) C-10 positions on pharmacological activity, this study constructed GB ester derivatives by the esterification reaction of GB as the parent nucleus with compounds with aromatic groups such as p-chlorobenzoic acid, p-fluorobenzoic acid, p-nitrobenzoic acid, p-methoxybenzoic acid, 3-methoxybenzoic acid, and 3'5'-dinitrobenzoic acid by reference to the design of nicotinic acid esters, cinnamic acid esters, and benzoic esters [22-30]. The GB derivatives were structurally characterized by nuclear magnetic resonance spectroscopy (NMR), tested for in vitro tumor proliferation inhibitory activity by thiazolyl blue (MTT), and detected for ovarian cancer SKOV3 cell apoptosis by the Annexin V/PI double staining assay.

\section{Materials And Methods}

\section{Chemistry}

All reagents and solvents used in this study were purchased from Tianjin Fuyu Fine Chemical Co., Ltd. (P.R. China), Shanghai Macklin Biochemical Technology Co., Ltd. (P.R. China), and Energy Chemical (P.R. China), and used as received without further purification unless otherwise noted. In addition, some biological reagents were purchased from Sigma or GIBCO, USA.

TLC was carried out on Silica Gel GF254 plates (Qingdao Haiyang Chemical Co., Ltd) and spots were visualized by iodine vapors or by irradiation with UV light $(254 \mathrm{~nm})$. All water employed was ultrapure (> 18.2 $\mathrm{M} \Omega \mathrm{cm}-1$ at $25^{\circ} \mathrm{C}$, Milli-Q, Millipore, Billerica, MA). The NMR spectra were measured by Bruker Avance 600 NMR analyzer (Bruker, Switzerland) in the indicated solvents. Chemical shifts are expressed in ppm ( $\delta$ units) relative to TMS signal as internal reference, and the coupling constant values $(J)$ are shown in Hertz. Signal multiplicities are reported by the following abbreviations: $s$ (singlet), $d$ (doublet), $t$ (triplet), dd (double doublet), q (quartet), $m$ (multiplet), and brs (broad signal). HB DAC-50 was used for preparative liquid chromatograph (Jiangsu Hanbon Science \& Technology Co., Ltd.). Agilent 1260 high performance liquid chromatograph (Agilent Technologies Co. Ltd, USA), SCIENTZ-10N vacuum freeze dryer (Ningbo Xinzhi Biotechnology Co. Ltd.), YG-875B ultra clean bench (Suzhou Medical Equipment Factory), inverted phase contrast microscope (Zeiss, Germany), enzyme marker (Biotek, USA), $\mathrm{CO}_{2}$ cell incubator (Thermo Fisher Scientific), and GB (98.24\% purity, lab-made) were used.

\section{Tumor cell growth inhibitory assay}

The SKOV3 cells used in the following cell experiments were obtained from the Shanghai EK-Bioscience Co., Ltd. (P.R. China). SKOV3 cells were maintained in DMEM containing 10\% fetal bovine serum (FBS). 
All cells were grown at $37^{\circ} \mathrm{C}$ in a humidified atmosphere of $5 \% \mathrm{CO}_{2}$. All the reagents used in this study, unless otherwise indicated, were purchased from Sigma (USA). The compounds with inhibitory activity against human ovarian cancer cells SKOV3 were screened by the MTT assay [31]. Briefly, SKOV3 cells were cultured at a density of $80 \%$ to $90 \%$ and inoculated in 96 -well plates at $2 \times 10^{4} /$ well. The attached cells were placed overnight and treated with increasing concentrations of drug for $24 \mathrm{~h}$. Blank controls (no drug treatment), and experiment groups (containing $5 \mu \mathrm{mol} / \mathrm{L}, 10 \mu \mathrm{mol} / \mathrm{L}, 20 \mu \mathrm{mol} / \mathrm{L}, 40 \mu \mathrm{mol} / \mathrm{L}, 60$ $\mu \mathrm{mol} / \mathrm{L}, 80 \mu \mathrm{mol} / \mathrm{L}$, and $100 \mu \mathrm{mol} / \mathrm{L}$ ) of ginkgolide $B$ derivatives compounds 1 to 11 were used to treat ovarian cancer cells, respectively. After $24 \mathrm{~h}$ of cell culture, $20 \mu \mathrm{L}$ of MTT was added to the cell medium of each well, and the plates were mixed by gently tapping. After continuing incubation for $4 \mathrm{~h}$, the culture supernatant in the wells was aspirated and discarded, $150 \mu \mathrm{L}$ of DMSO solution was added to each well and shaken for $10 \mathrm{~min}$ on a shaker. Cell growth was examined by detecting the absorbance value (A) of each well at a wavelength of $550 \mathrm{~nm}$ by an enzyme marker to preliminarily investigate the optimal concentration of compounds 1 11 on the viability of SKOV3 cells. Three replicate wells were set up for each concentration. The inhibition rate was calculated according to the following formula, and $\mathrm{IC}_{50}$ was calculated using the software Prism 5 (GraphPad Software, Inc.).

Inhibition rate $=[1-(O D$ of treatment $-O D$ of blank $) /(O D$ of control $-O D$ of blank $)] \times 100 \%$

$\lg I C_{50}=X m-I[P-(3-P m-P n) / 4]$

$\mathrm{Xm}$ - Ig Maximum dose

I- $\lg$ (maximum dose/adjacent dose)

$P-$ (sum of positive rate)

$P m$ - (Maximum positive rate)

$P n-($ minimum positive rate)

\section{Annexin V/PI double staining assay to observe SKOV3 cell apoptosis}

Annexin V-FITC Apoptosis Detection Kit was purchased from Sigma (USA) and used according to the manufacturer's instructions. Human ovarian cancer cells SKOV3 at the logarithmic growth stage were inoculated in 6-well plates at $37^{\circ} \mathrm{C}, 5 \% \mathrm{CO}_{2}$, and cultured overnight. Drug-treated groups were treated with compound $\mathbf{1}, \mathbf{3}, \mathbf{6}$, and 10 at the optimal mass concentration for $48 \mathrm{~h}$. The negative control group was treated with McCoy's 5A complete medium only. After digestion of cells with EDTA-free trypsin, cells were collected by centrifugation at $4{ }^{\circ} \mathrm{C}, 7000 \mathrm{rpm} / \mathrm{min}$ for $5 \mathrm{~min}$. Cells were resuspended in precooled sterilized PBS at $4^{\circ} \mathrm{C}$ and $7000 \mathrm{rpm} / \mathrm{min}$ for $5 \mathrm{~min}$, and the supernatant was discarded. The $1 \times$ binding buffer $(195 \mu \mathrm{L})$ was added. After suspending the cells, $5 \mu \mathrm{L}$ of Annexin V-FITC was added and mixed gently. Ten $\mu \mathrm{L}$ of propidium iodide staining solution was added, mixed gently, and incubated at room temperature for $15 \mathrm{~min}$ protected from light. Stained samples were analyzed immediately by a FACScan 
flow cytometer (Beckman coulter EPICS xL). The fractions of cell population in different quadrants were analyzed using quadrant statistics. Cells in the lower right quadrant represented apoptosis while cells in the upper right quadrant represented necrosis or post apoptotic necrosis.

\section{General procedure for the synthesis of GB derivatives 1-11}

The synthetic route was shown in Fig. 1. A $100.0 \mathrm{~mL}$ round bottom flask was added with $212.2 \mathrm{mg}$ of GB $(0.5 \mathrm{mmol}), 122.2 \mathrm{mg}$ of 4-dimethylaminopyridine (DMAP) $(1.0 \mathrm{mmol}), 191.7 \mathrm{mg}$ of 1-ethyl-carbodiimide hydrochloride (EDC-HCl) $(1.0 \mathrm{mmol})$ and 6 benzoic acids ( $p$-chlorobenzoic acid, $p$-fluorobenzoic acid, $p$ nitrobenzoic acid, $p$-methoxybenzoic acid, 3-methoxybenzoic acid, 3,5-dinitrobenzoic acid) with $1.0 \mathrm{mmol}$, respectively. Anhydrous dichloromethane $50.0 \mathrm{~mL}$ was added and stirred under room temperature. The reaction was detected by TLC after $6 \mathrm{~h}$ at $1 \mathrm{~h}$ intervals and fumigated with sulfuric acid-ethanol solution or acetic acid-iodine. The reaction was considered complete when the spots of GB disappeared. After recovering the dichloromethane solution under reduced pressure, the residue was dissolved with $30.0 \mathrm{~mL}$ of ethyl acetate and underwent reverse extraction three times with water, with $15.0 \mathrm{~mL} /$ time. The ethyl acetate layer was dried by anhydrous sodium sulfate and concentrated under reduced pressure to dryness. The product of each synthesis was dissolved in acetonitrile to make a sample solution of 30.0 $\mathrm{mg} / \mathrm{mL}$. After passing through a $0.45 \mu \mathrm{m}$ filter membrane, the product was purified by high pressure preparative chromatography eluting with acetonitrile/water to produce the corresponding derivatives.

\section{1-(4-chlorobenzoyl) GB (1)}

White powder, yield 12.12\%, $\lambda_{\max }=254 \mathrm{~nm} .{ }^{1} \mathrm{H} \mathrm{NMR}\left(600 \mathrm{MHz}\right.$, DMSO- $\left.d_{6}\right) \delta 8.04(\mathrm{~m}, 2 \mathrm{H}), 7.65(\mathrm{dd}, \mathrm{J}=$ $11.2,4.4 \mathrm{~Hz}, 2 \mathrm{H}), 6.71(\mathrm{~d}, \mathrm{~J}=4.9 \mathrm{~Hz}, 1 \mathrm{H}), 6.67(\mathrm{~s}, 1 \mathrm{H}), 6.16(\mathrm{~d}, \mathrm{~J}=93.6 \mathrm{~Hz}, 1 \mathrm{H}), 5.69(\mathrm{dd}, \mathrm{J}=61.2,3.8 \mathrm{~Hz}$, $1 \mathrm{H}), 5.64(\mathrm{~d}, \mathrm{~J}=3.6 \mathrm{~Hz}, 1 \mathrm{H}), 4.96(\mathrm{~m}, 1 \mathrm{H}), 4.93(\mathrm{~d}, \mathrm{~J}=5.6 \mathrm{~Hz}, 1 \mathrm{H}), 2.97(\mathrm{~m}, 1 \mathrm{H}), 2.22(\mathrm{dd}, \mathrm{J}=13.6,4.5 \mathrm{~Hz}$, 1H), 2.00 (ddd, J = 36.1, 28.3, 24.2 Hz, 1H), 1.79 (ddd, J = 31.8, 14.3, 4.4 Hz, 1H), 1.13 (dd, J = 28.9, $5.1 \mathrm{~Hz}$, $3 \mathrm{H}), 1.01$ (d, J = 6.7 Hz, 9H); ${ }^{13} \mathrm{C}$ NMR (151 MHz, DMSO-d 6 ) $\delta 176.45,173.78,170.41,163.44,139.07$, $131.89,129.51,128.51,109.59,99.31,91.71,83.85,79.84,75.40,71.04,68.72,68.30,48.88,41.70,40.54$, 37.07, 32.45, 29.35, 8.62. ESI-MS: $m / z 563.3[\mathrm{M}+\mathrm{H}]^{+}$.

\section{0-(4-chlorobenzoyl) GB (2)}

White powder, $15.48 \%$ yield, $\lambda_{\max }=254 \mathrm{~nm} .{ }^{1} \mathrm{H}$ NMR $\left(600 \mathrm{MHz}\right.$, DMSO- $\left.d_{6}\right) \delta 8.06(\mathrm{~m}, 2 \mathrm{H}), 7.54(\mathrm{~m}, 2 \mathrm{H})$, $6.82(\mathrm{~s}, 1 \mathrm{H}), 6.48(\mathrm{~s}, 1 \mathrm{H}), 6.25(\mathrm{~s}, 1 \mathrm{H}), 6.22(\mathrm{~s}, 1 \mathrm{H}), 5.46(\mathrm{~s}, 1 \mathrm{H}), 4.62(\mathrm{t}, J=21.1 \mathrm{~Hz}, 1 \mathrm{H}), 4.09(\mathrm{~d}, J=5.3 \mathrm{~Hz}$, $1 \mathrm{H}), 2.86(\mathrm{q}, J=7.0 \mathrm{~Hz}, 1 \mathrm{H}), 2.14(\mathrm{~m}, 1 \mathrm{H}), 1.75(\mathrm{~d}, J=12.6 \mathrm{~Hz}, 2 \mathrm{H}), 1.07(\mathrm{~d}, J=7.2 \mathrm{~Hz}, 3 \mathrm{H}), 0.90(\mathrm{~s}, 9 \mathrm{H}, \mathrm{t}-$ $\mathrm{Bu}) ;{ }^{13} \mathrm{C}$ NMR $\left(151 \mathrm{MHz}\right.$, DMSO- $\left.d_{6}\right) \delta 176.72,170.41,168.96,164.18,139.94,132.47,129.55,127.05$, $110.46,100.16,94.36,83.33,78.66,74.38,72.38,70.72,67.45,48.89,41.88,40.53,37.22,32.21,30.57$, 28.89, 8.51. ESI-MS: $m / z 563.3[\mathrm{M}+\mathrm{H}]^{+}$.

\section{1-(4-fluorobenzoyl) GB (3)}


White powder, yield 15.13\%, $\lambda_{\max }=210 \mathrm{~nm},{ }^{1} \mathrm{H}$ NMR $\left(600 \mathrm{MHz}\right.$, DMSO- $\left.d_{6}\right) \delta 8.00(\mathrm{~m}, 2 \mathrm{H}), 7.32(\mathrm{~m}, 2 \mathrm{H})$, $6.64(\mathrm{t}, \mathrm{J}=4.3 \mathrm{~Hz}, 1 \mathrm{H}), 6.61(\mathrm{~s}, 1 \mathrm{H}), 6.01(\mathrm{~s}, 1 \mathrm{H}), 5.68(\mathrm{~d}, \mathrm{~J}=4.1 \mathrm{~Hz}, 1 \mathrm{H}), 5.46(\mathrm{dd}, \mathrm{J}=14.8,5.1 \mathrm{~Hz}, 1 \mathrm{H})$, $4.91(\mathrm{~d}, \mathrm{~J}=6.4 \mathrm{~Hz}, 1 \mathrm{H}), 4.84(\mathrm{~m}, 1 \mathrm{H}), 2.91(\mathrm{~m}, 1 \mathrm{H}), 2.14(\mathrm{~m}, 1 \mathrm{H}), 1.98(\mathrm{~m}, 1 \mathrm{H}), 1.71(\mathrm{~m}, 1 \mathrm{H}), 1.07(\mathrm{~m}, 3 \mathrm{H})$, $0.92(\mathrm{~m}, 9 \mathrm{H}) ;{ }^{13} \mathrm{C}$ NMR $\left(151 \mathrm{MHz}, \mathrm{DMSO}-d_{6}\right) \delta 176.46,173.78,170.44,166.64,165.18,163.31,133.00$, $126.30,116.54$, 109.58, 99.36 , 91.78, 83.85, 79.86, 75.31, $70.10,69.14,68.31$, 48.88, 41.69 , 40.48 , $37.07,32.45,29.35,8.64$. ESI-MS: $m / z 547.2[\mathrm{M}+\mathrm{H}]^{+}$.

\section{0-(4-fluorobenzoyl) GB (4)}

White powder, $36.47 \%$ yield, $\lambda_{\max }=210 \mathrm{~nm},{ }^{1} \mathrm{H}$ NMR $\left(600 \mathrm{MHz}, \mathrm{DMSO}-d_{6}\right) \delta 8.21(\mathrm{~m}, 2 \mathrm{H}), 7.32(\mathrm{t}, \mathrm{J}=8.8$ $\mathrm{Hz}, 2 \mathrm{H}), 6.82(\mathrm{~d}, \mathrm{~J}=4.9 \mathrm{~Hz}, 1 \mathrm{H}), 6.47(\mathrm{~s}, 1 \mathrm{H}), 6.25(\mathrm{~s}, 1 \mathrm{H}), 6.22(\mathrm{~s}, 1 \mathrm{H}), 5.46(\mathrm{~d}, \mathrm{~J}=2.9 \mathrm{~Hz}, 1 \mathrm{H}), 4.64(\mathrm{~d}, \mathrm{~J}=$ $6.4 \mathrm{~Hz}, 1 \mathrm{H}), 4.09(\mathrm{dd}, \mathrm{J}=6.3,5.1 \mathrm{~Hz}) 6.3,5.1 \mathrm{~Hz}, 1 \mathrm{H}), 2.87(\mathrm{q}, \mathrm{J}=7.1 \mathrm{~Hz}, 1 \mathrm{H}), 2.14(\mathrm{~m}, 1 \mathrm{H}), 1.77(\mathrm{~m}, 2 \mathrm{H})$, 1.07 (m, 3H), 0.90 (s, 9H,t-Bu); ${ }^{13} \mathrm{C}$ NMR (151 MHz, DMSO-d $\left.d_{6}\right) \delta 176.72,170.42,169.03,165.41,164.00$, $133.74,133.68,124.83,116.62,110.84,100.16,94.39,83.35,78.66,74.40,72.39,70.59,67.47,48.89$, $41.88,40.48,37.23,32.21,28.89,8.51$. ESI-MS: $m / z$ 547.2 [M+H] $]^{+}$.

\section{1-(4-methoxybenzoyl) GB (5)}

White powder, yield 8.40\%, molecular formula: $\mathrm{C}_{28} \mathrm{H}_{30} \mathrm{O}_{12}, \lambda_{\max }=254 \mathrm{~nm},{ }^{1} \mathrm{H}$ NMR $(600 \mathrm{MHz}, \mathrm{DMSO}-$ $\left.d_{6}\right) \delta 7.93(\mathrm{dd}, \mathrm{J}=12.1,8.8 \mathrm{~Hz}, 2 \mathrm{H}), 7.08(\mathrm{dd}, \mathrm{J}=8.8,3.8 \mathrm{~Hz}, 2 \mathrm{H}), 6.66(\mathrm{~m}, 1 \mathrm{H}), 6.07(\mathrm{~s}, 1 \mathrm{H}), 5.93(\mathrm{~m}, 1 \mathrm{H})$, $5.74(\mathrm{~m}, 1 \mathrm{H}), 5.52(\mathrm{dd}, \mathrm{J}=14.8,4.9 \mathrm{~Hz}, 1 \mathrm{H}), 4.90(\mathrm{~m}, 1 \mathrm{H}), 3.85(\mathrm{~d}, \mathrm{~J}=5.6 \mathrm{~Hz}, 3 \mathrm{H}), 2.92(\mathrm{~m}, 1 \mathrm{H}), 2.21(\mathrm{dd}, \mathrm{J}$ $=12.2,8.1 \mathrm{~Hz}, 1 \mathrm{H}), 2.05(\mathrm{~m}, 1 \mathrm{H}), 1.92(\mathrm{td}, \mathrm{J}=13.6,3.7 \mathrm{~Hz}, 1 \mathrm{H}), 1.79(\mathrm{~m}, 1 \mathrm{H}), 1.27(\mathrm{~m}, 2 \mathrm{H}), 1.16(\mathrm{~d}, \mathrm{~J}=7.2$ $\mathrm{Hz}, 1 \mathrm{H}), 0.98$ (s, 9H,t-Bu); ${ }^{13} \mathrm{C}$ NMR $\left(151 \mathrm{MHz}, \mathrm{DMSO}-d_{6}\right) \delta 176.82,169.76,164.33,163.83,131.98,121.01$, $114.54,110.03,108.64,104.27,98.48,85.44,83.90,80.24,73.23,70.20,68.88,68.44,56.03,49.92,48.38$, $42.03,36.72,31.48,29.45,8.72$. ESI-MS: $m / z 559.3[\mathrm{M}+\mathrm{H}]^{+}$.

\section{0-(4-methoxybenzoyl) GB (6)}

White powder, yield $55.57 \%, \lambda_{\max }=254 \mathrm{~nm},{ }^{1} \mathrm{H}$ NMR $\left(600 \mathrm{MHz}, \mathrm{DMSO}-d_{6}\right) \delta 8.15(\mathrm{dd}, \mathrm{J}=16.9,8.8 \mathrm{~Hz}$, 2H), $7.08(\mathrm{~m}, 2 \mathrm{H}), 6.80(\mathrm{t}, \mathrm{J}=47.4 \mathrm{~Hz}, 1 \mathrm{H}), 6.53(\mathrm{~s}, 1 \mathrm{H}), 6.23(\mathrm{~m}, 1 \mathrm{H}), 5.52(\mathrm{~m}, 1 \mathrm{H}), 4.65(\mathrm{dd}, \mathrm{J}=50.4,15.3$ $\mathrm{Hz}, 1 \mathrm{H}), 4.16(\mathrm{dd}, \mathrm{J}=41.0,35.2 \mathrm{~Hz}, 1 \mathrm{H}), 3.79(\mathrm{~m}, 3 \mathrm{H}), 2.93(\mathrm{~m}, 1 \mathrm{H}), 2.17(\mathrm{~m}, 1 \mathrm{H}), 1.80(\mathrm{~m}, 1 \mathrm{H}), 1.13(\mathrm{~m}$, 4H), 1.03 (dd, $\mathrm{J}=16.2,9.1 \mathrm{~Hz}, 1 \mathrm{H}), 0.96(\mathrm{~s}, 9 \mathrm{H}, \mathrm{t}-\mathrm{Bu}) ;{ }^{13} \mathrm{C} \mathrm{NMR}\left(151 \mathrm{MHz}, \mathrm{DMSO}-d_{6}\right) \delta 176.74,169.88$, $169.25,164.54,132.92,131.08,120.43,114.64,110.35,100.27,94.51,83.44,78.66,74.46,72.48,70.16$, $67.52,56.04,48.91,41.85,40.48,37.17,32.19,30.61,28.90,8.60$. ESI-MS: $m / z 559.3[\mathrm{M}+\mathrm{H}]^{+}$.

\section{1-(4-Nitrobenzoyl) GB (7)}

White powder, $38.62 \%$ yield, $\lambda_{\max }=254 \mathrm{~nm},{ }^{1} \mathrm{H} \mathrm{NMR}\left(600 \mathrm{MHz}\right.$, DMSO- $\left.d_{6}\right) \delta 8.38(\mathrm{~m}, 2 \mathrm{H}), 8.24(\mathrm{~m}, 2 \mathrm{H})$, $6.72(\mathrm{~m}, 1 \mathrm{H}), 6.09(\mathrm{~d}, \mathrm{~J}=11.5 \mathrm{~Hz}, 1 \mathrm{H}), 5.78(\mathrm{t}, \mathrm{J}=11.0 \mathrm{~Hz}, 1 \mathrm{H}), 5.53(\mathrm{~m}, 1 \mathrm{H}), 5.05(\mathrm{t}, \mathrm{J}=11.1 \mathrm{~Hz}, 1 \mathrm{H}), 4.93$ (m, 1H), $2.98(\mathrm{~m}, 1 \mathrm{H}), 2.24(\mathrm{~m}, 1 \mathrm{H}), 2.03(\mathrm{~m}, 1 \mathrm{H}), 1.78(\mathrm{~m}, 1 \mathrm{H}), 1.28(\mathrm{~m}, 1 \mathrm{H}), 1.14(\mathrm{~m}, 3 \mathrm{H}), 1.01(\mathrm{~s}, 9 \mathrm{H}, \mathrm{t}-\mathrm{Bu})$; 
${ }^{13} \mathrm{C}$ NMR $\left(151 \mathrm{MHz}\right.$, DMSO- $\left.d_{6}\right) \delta 176.44,173.81,170.33,162.89,150.94,134.95,131.44,124.39,109.66$, $99.24,91.52,83.85,79.83,75.87,70.99,69.17,68.30,48.87,41.73,40.55,37.12,32.49,29.34,14.07,8.56$. ESI-MS: $m / z 574.4[\mathrm{M}+\mathrm{H}]^{+}$.

\section{0-(4-Nitrobenzoyl) GB (8)}

White powder, $26.1 \%$ yield, $\lambda_{\max }=254 \mathrm{~nm},{ }^{1} \mathrm{H}$ NMR $\left(600 \mathrm{MHz}, \mathrm{DMSO}-\mathrm{d}_{6}\right) \delta 8.38(\mathrm{~m}, 2 \mathrm{H}), 8.23(\mathrm{dd}, \mathrm{J}=$ 18.7, 8.7 Hz, 2H), $6.77(\mathrm{~d}, \mathrm{~J}=4.7 \mathrm{~Hz}, 1 \mathrm{H}), 6.07(\mathrm{~m}, 1 \mathrm{H}), 5.74(\mathrm{t}, \mathrm{J}=30.6 \mathrm{~Hz}, 1 \mathrm{H}), 5.55(\mathrm{~m}, 1 \mathrm{H}), 5.05(\mathrm{t}, \mathrm{J}=$ $11.1 \mathrm{~Hz}, 1 \mathrm{H}), 4.91(\mathrm{dd}, \mathrm{J}=34.0,4.7 \mathrm{~Hz} 34.0,4.7 \mathrm{~Hz}, 1 \mathrm{H}), 2.92(\mathrm{~m}, 1 \mathrm{H}), 2.24(\mathrm{~m}, 1 \mathrm{H}), 2.05(\mathrm{~m}, 1 \mathrm{H}), 1.79(\mathrm{~m}$, 1H), 1.28 (ddd, J = 60.3, 40.8, 20.6 Hz, 1H), $1.18(\mathrm{~s}, 3 \mathrm{H}), 1.01$ (s, 9H,t-Bu); ${ }^{13} \mathrm{C}$ NMR (151 MHz, DMSO$\left.d_{6}\right) \delta 176.44,173.81,170.33,162.89,150.94,134.96,131.53,124.43,110.88,99.25,91.52,83.85,80.54$, 75.87, 70.99, 68.74, 68.30, 48.88, 41.72, 40.50, 37.12, 32.49, 30.79, 29.34, 8.56. ESI-MS: $m / z$ 574.4 $[\mathrm{M}+\mathrm{H}]^{+}$.

\section{1-(5-methoxybenzoyl) GB (9)}

White powder, yield 8.72\%, $\lambda_{\max }=254 \mathrm{~nm},{ }^{1} \mathrm{H}$ NMR $\left(600 \mathrm{MHz}, \mathrm{DMSO}-d_{6}\right) \delta 7.78$ (ddd, $\mathrm{J}=13.3,4.6,3.6 \mathrm{~Hz}$, 2H), $7.47(\mathrm{~m}, 2 \mathrm{H}), 6.82(\mathrm{t}, \mathrm{J}=41.7 \mathrm{~Hz}, 1 \mathrm{H}), 6.52(\mathrm{~d}, \mathrm{~J}=45.7 \mathrm{~Hz}, 1 \mathrm{H}), 6.31(\mathrm{~s}, 1 \mathrm{H}), 6.29(\mathrm{~s}, 1 \mathrm{H}), 5.51(\mathrm{~m}$, $1 \mathrm{H}), 4.64(\mathrm{~m}, 1 \mathrm{H}), 4.42(\mathrm{~m}, 1 \mathrm{H}), 4.17(\mathrm{dt}, \mathrm{J}=22.0,11.0 \mathrm{~Hz}, 1 \mathrm{H}), 3.80(\mathrm{~m}, 1 \mathrm{H}), 2.88(\mathrm{~m}, 1 \mathrm{H}), 2.21(\mathrm{~m}, 1 \mathrm{H})$, $1.85(\mathrm{~m}, 1 \mathrm{H}), 1.14(\mathrm{~d}, \mathrm{~J}=7.3 \mathrm{~Hz}, 1 \mathrm{H}), 1.03(\mathrm{~m}, 4 \mathrm{H}), 0.96(\mathrm{~s}, 9 \mathrm{H}, \mathrm{t}-\mathrm{Bu}) ;{ }^{13} \mathrm{C}$ NMR $(151 \mathrm{MHz}$, DMSO$\left.d_{6}\right) \delta 177.64,169.76,169.06,165.10,159.72,130.23,129.47,122.12,114.87,110.40,100.19,94.27$, $83.60,79.06,74.39,72.59,70.54,67.89,55.85,48.89,42.27,33.73,31.75,30.61,29.12,8.43$. ESI-MS: $m / z$ $559.3[\mathrm{M}+\mathrm{H}]^{+}$.

\section{0-(5-methoxybenzoyl) GB (10)}

White powder, yield 38.90\%, $\lambda_{\max }=254 \mathrm{~nm},{ }^{1} \mathrm{H}$ NMR $\left(600 \mathrm{MHz}, \mathrm{DMSO}-d_{6}\right) \delta 7.56(\mathrm{~m}, 1 \mathrm{H}), 7.49(\mathrm{~m}, 2 \mathrm{H})$, $7.27(\mathrm{~m}, 1 \mathrm{H}), 6.69(\mathrm{dt}, \mathrm{J}=35.6,19.0 \mathrm{~Hz}, 1 \mathrm{H}), 6.06(\mathrm{~m}, 1 \mathrm{H}), 5.76(\mathrm{~m}, 1 \mathrm{H}), 5.56(\mathrm{ddd}, \mathrm{J}=31.9,19.4,5.0 \mathrm{~Hz}$, 1H), $5.02(\mathrm{dd}, \mathrm{J}=36.5,17.5 \mathrm{~Hz}, 1 \mathrm{H}), 4.89(\mathrm{dd}, \mathrm{J}=33.1,5.1 \mathrm{~Hz}, 1 \mathrm{H}), 4.08(\mathrm{~s}, 1 \mathrm{H}), 3.87(\mathrm{~m}, 3 \mathrm{H}), 2.96(\mathrm{~m}, 1 \mathrm{H})$, $2.25(\mathrm{~m}, 1 \mathrm{H}), 2.01(\mathrm{~m}, 1 \mathrm{H}), 1.81(\mathrm{~m}, 1 \mathrm{H}), 1.23(\mathrm{~m}, 1 \mathrm{H}), 1.18(\mathrm{~m}, 2 \mathrm{H}), 0.99(\mathrm{~s}, 9 \mathrm{H}, \mathrm{t}-\mathrm{Bu}) ;{ }^{13} \mathrm{C}$ NMR $(151 \mathrm{MHz}$, DMSO- $\left.d_{6}\right) \delta 176.48,173.38,170.44,164.25,159.74,131.14,122.09,119.67,114.76,109.59,99.26,91.79$, $83.46,79.49,76.46,73.32,71.06,68.50,55.83,49.40,40.89,36.97,32.49,29.34,13.16,8.60$. ESI-MS: $\mathrm{m} / z$ $559.3[\mathrm{M}+\mathrm{H}]^{+}$.

\section{1-(3-5-Dinitrobenzoyl) GB (11)}

White powder, 61.25\% yield, $\lambda_{\max }=254 \mathrm{~nm},{ }^{1} \mathrm{H} \mathrm{NMR}\left(600 \mathrm{MHz}\right.$, DMSO- $\left.d_{6}\right) \delta 9.06(\mathrm{~m}, 1 \mathrm{H}), 8.98(\mathrm{dd}, \mathrm{J}=$ 15.0, $2.1 \mathrm{~Hz}, 2 \mathrm{H}), 6.82(\mathrm{~m}, 2 \mathrm{H}), 6.12(\mathrm{~s}, 1 \mathrm{H}), 5.86(\mathrm{~d}, \mathrm{~J}=4.1 \mathrm{~Hz}, 1 \mathrm{H}), 5.53(\mathrm{dd}, \mathrm{J}=30.6,5.3 \mathrm{~Hz}, 1 \mathrm{H}), 5.21(\mathrm{~d}$, $\mathrm{J}=6.9 \mathrm{~Hz}, 1 \mathrm{H}), 4.93(\mathrm{~m}, 1 \mathrm{H}), 2.98(\mathrm{~m}, 1 \mathrm{H}), 2.24(\mathrm{dt}, \mathrm{J}=41.8,21.0 \mathrm{~Hz}, 1 \mathrm{H}), 1.97(\mathrm{~m}, 1 \mathrm{H}), 1.73(\mathrm{~m}, 1 \mathrm{H}), 1.13$ (m, 3H), $0.99(\mathrm{~m}, 9 \mathrm{H}) ;{ }^{13} \mathrm{C}$ NMR (151 MHz, DMSO-d 6 ) $\delta 176.43,173.80,170.22,161.54,148.97,132.60$, 
$130.40,129.27,123.39,109.78,99.04,91.18,83.58,79.67,76.52,70.86,69.22,68.25,48.86,41.76,40.53$, 36.94, 30.88, 29.30, 8.37. ESI-MS: $m / z 619.4[\mathrm{M}+\mathrm{H}]^{+}$.

\section{Results And Discussion}

According to the reaction described in Scheme 1, eleven novel GB derivatives 1-11 were synthesized by esterification using GB as a raw material. Two products can be simultaneously obtained in one pot reaction. Due to the higher reaction activity of C-10 hydroxyl in GB, the yields of esterification at the C-10 position were higher than at the C-1 position. Conformational analysis showed that compounds 1, 3, 5, 7, 9 and 11 were hydroxyesterification products at the $\mathrm{C}-1$ position of $\mathrm{GB}$, and compounds $2,4,6,8$, and 10 were hydroxyesterification products at the $\mathrm{C}-10$ position of GB. A method for the rapid separation and purification of GB derivatives by preparative chromatography was established. The structure of all the final compounds 1-11 was confirmed by ${ }^{1} \mathrm{H}$ NMR and ${ }^{13} \mathrm{C}$ NMR. For the NMR spectra of compounds 111 , a singlet at $\delta$ 1.0-0.9 ppm that corresponded to tert butyl functionality, signals at $\delta$ 9.1-6.0 ppm revealed the presence of the benzene ring.

On the basis of previous studies, the final products 1-11 and GB were evaluated for their in vitro antiproliferative activity against ovarian cancer SKOV3 cell lines by MTT assay. The proliferation inhibitory activity of these compounds was measured at different concentrations. The $\mathrm{IC}_{50}$ values for each sample were obtained by plotting the inhibition rate against the drug concentration. The results are presented in Table 1. As we defined that compounds showing less than $50 \%$ inhibitory rate at $100 \mu \mathrm{M}$ were inactive. Compounds $3,4,7,8$ and 11 hardly showed any activity, the $\mathrm{IC}_{50}$ values against the SKOV3 cell lines are all above $100 \mu \mathrm{M}$. The rest of the compounds showed moderate to good activity on SKOV3 cell lines with $\mathrm{IC}_{50}$ values $15.65-63.30 \mu \mathrm{M}$. Obviously, as compared with compound 1 and 2 , the esterification position of $\mathrm{C}-1$ or $\mathrm{C}-10$ has little effect on the activity of the compounds. However, the inhibitory activities were increased by introduce of the electron-donating group into the benzyl group.

Table 1

In vitro antiproliferative activity of $\mathrm{GB}$ derivatives. $\mathrm{IC}_{50}$ values are shown in $\mu \mathrm{M}$ against SKOV3 cell line, respectively.

\begin{tabular}{|llll|}
\hline Compounds & $\mathbf{I C}_{\mathbf{5 0}}$ & Compounds & $\mathbf{I C}_{\mathbf{5 0}}$ \\
\hline $\mathrm{GB}$ & $45.4 \pm 1.24$ & 6 & $63.30 \pm 2.04$ \\
\hline 1 & $16.05 \pm 086$ & 7 & $>100$ \\
\hline 2 & $15.65 \pm 0.78$ & 8 & $>100$ \\
\hline 3 & $>100$ & 9 & $23.20 \pm 0.97$ \\
4 & $>100$ & 10 & $31.10 \pm 1.05$ \\
\hline 5 & $32.00 \pm 1.12$ & 11 & $>100$ \\
\hline & & & \\
\hline
\end{tabular}


The apoptosis of ovarian cancer SKOV3 cells was induced by $100 \mu \mathrm{mol} / \mathrm{L}$ of GB, compounds 2, 5, and 9. The apoptosis rates were found to be $7.98 \%, 31.68 \%, 8.82 \%$, and $9.46 \%$, respectively. Preliminary in vitro anti-tumor cell proliferation activity test and the Annexin V/PI double staining assay showed that compound $\mathbf{2}$ had the highest inhibition rate against ovarian cancer SKOV3 cells and could inhibit the proliferation of tumor cells and induce apoptosis. Ginkgolide B derivatives hydroxyesterified at the C-10 position showed significantly higher apoptosis rates than the hydroxyesterified product at the $\mathrm{C}-1$ position in ovarian cancer cells SKOV3.

\section{Conclusion}

In this study, 11 novel GB derivatives 1-11 were synthesized and evaluated for their in vitro antiproliferative activities against human ovarian cancer SKOV3 cell lines. The majority of the compounds showed moderate to good activity on SKOV3 cell lines. Among these derivatives, compound 2 was found to exhibit better cytotoxic activity on against SKOV3 cells with $\mathrm{IC}_{50}$ values $15.65 \mu \mathrm{M}$. Annexin V/PI double staining assay showed that compound 2 induced apoptosis in SKOV3 cells to a slightly greater extent than GB and compounds $\mathbf{5}$, and $\mathbf{9}$, with an apoptosis rate of $31.68 \%$. The above findings will be of great significance for the development of GB derivatives as potential antitumor agents.

\section{Declarations}

\section{Acknowledgements}

This work was supported by the National Natural Science Foundation of China (31702206), Key projects of science and Technology Department of Shaanxi Province (2018ZDXM-SF-083), and Natural Science Foundation of Shaanxi Province of China (2019JQ-530).

\section{Conflict of interest}

The authors declare that they have no known competing financial interests or personal relationships that could have appeared to influence the work reported in this paper.

\section{References}

1. Ahlemeyer B, Krieglstein J (2003) Pharmacological studies supporting the therapeutic use of Ginkgo biloba extract for Alzheimer's disease. Pharmacopsychiatry 36(1):8-14

2. Bate C, Salmona M, Williams A (2004) Ginkgolide B inhibits the neurotoxicity of prions or amyloidbeta1-42. J Neuroinflamm 1(1):4

3. Tian QY, Gong LL (2016) Research progress of ginkgolide. Zhongnan Pharmacol 14(8):838-841

4. Nabavi SM, Habtemariam S, Daglia M, Braidy N, Loizzo MR, Tundis R, Nabavi SF (2015) Neuroprotective Effects of Ginkgolide B Against Ischemic Stroke: A Review of Current Literature. Curr Top Med Chem 15(21):2222-2232 
5. Pan M, Yuan Y, Hui A-L et al (2012) Anti-platelet aggregation activity of ginkgolide B precursor drugs. Chin J Pharmacol 28(10):1435-1438

6. Huaian W, Jinsheng G (2017) Research progress of ginkgolide B for the treatment of malignant tumors. Guangming TCM 32(17):2589-2590

7. Gao T, Zhao R, Yao L, et al (2020) Platelet-activating factor induces the stemness of ovarian cancer cells via the PAF/PAFR signaling pathway[J]. American Journal of Translational Research 12(11):7249-7261

8. Jiang W, Cong Q, Wang Y, Ye B, Xu C (2014) Ginkgo may sensitize ovarian cancer cells to cisplatin: antiproliferative and apoptosis-inducing effects of ginkgolide $B$ on ovarian cancer cells. Integr Cancer Ther 13(3):NP10-N7

9. Chan W-H (2007) The signaling cascades of ginkgolide B-induced apoptosis in MCF-7 breast cancer cells. Int J Mol Sci 8(11):1177-1195

10. Yang B, Shuan S, Shengqiong C et al (2015) Study of ginkgolide B induced apoptosis in human colorectal cancer HCT116 cells. J Chengdu Med Coll 10(3):281-286

11. Zhi Y, Pan J, Shen W, He P, Zheng J, Zhou X et al (2016) Ginkgolide B inhibits human bladder cancer cell migration and invasion through MicroRNA-223-3p. Cell Physiol Biochem 39(5):1787-1794

12. Chan WH, Hsuuw YD (2007) Dosage effects of ginkgolide $B$ on ethanol-induced cell death in human hepatoma G2 cells. Ann NY Acad Sci 1095:388-398

13. Lou C, Lu H, Ma Z, Liu C, Zhang Y (2019) Ginkgolide B enhances gemcitabine sensitivity in pancreatic cancer cell lines via inhibiting PAFR/NF-KB pathway. Biomed Pharmacother 109:563-572

14. Yao B, Liu B, Shi L, Li X, Ren C, Cai M et al (2017) PAFR selectively mediates radioresistance and irradiation-induced autophagy suppression in prostate cancer cells. Oncotarget 8(8):13846-13854

15. Coyne CP, Narayanan L (2019) Carnosic Acid, Tangeretin, and ginkgolide-B anti-neoplastic cytotoxicity in dual combination with dexamethasone-[anti-EGFR] in pulmonary adenocarcinoma (A549). Anti Cancer Agents Med Chem 19(6):802-819

16. Sahu RP, Turner MJ, DaSilva SC, Rashid BM, Ocana JA, Perkins SM et al (2012) The environmental stressor ultraviolet $B$ radiation inhibits murine antitumor immunity through its ability to generate platelet-activating factor agonists. Carcinogenesis 33(7):1360-1367

17. Zhang L, Wang D, Jiang W et al (2010) Activated networking of platelet activating factor receptor and FAK/STAT1 induces malignant potential in BRCA1-mutant at-risk ovarian epithelium. BioMed Central 8(1):74

18. Aponte M, Jiang W, Lakkis M, Li MJ, Edwards D, Albitar L et al (2008) Activation of platelet-activating factor receptor and pleiotropic effects on tyrosine phospho-EGFR/Src/FAK/paxillin in ovarian cancers. Cancer Res 68(14):5839-5848

19. Yu Y, Zhang X, Hong S, Zhang M, Cai Q, Zhang M et al (2014) The expression of platelet-activating factor receptor modulates the cisplatin sensitivity of ovarian cancer cells: a novel target for combination therapy. Br J Cancer 111(3):515-524 
20. Chen Q, Chen L, Sun JB. Research progress on the sources, structural modifications and new dosage forms of ginkgolide compounds. J Nanjing. 2019 University of Traditional Chinese Medicine;35(3):344-50

21. Hui A-L, Wu Z-Y, Yuan Y, Zhou A, Pan M (2013) Synthesis of ginkgolide derivatives and analogs and progress in the study of PAFR and GlyR antagonistic activities. J Org Chem 33(6):1263-1272

22. Liu KZ, Xue LP, Xu YF et al (2018) Review and comparative analysis of benoxate synthesis processes. Henan Sci 36(09):1362-1366

23. Highest L, Jia L, Zhang Yin $Y$ et al (2016) Optimization of experimental reaction conditions for the synthesis of benoxate. J Xiangnan Coll 37(05):18-21

24. Wang Y (2014) Synthesis and characterization of benzoate. Exp Sci Technol 12(04):32-34

25. Dai GX, Wang L, Shoutao C (2014) Study on the catalytic synthesis process of benzoate. Coal Chem Ind 37(08):79-81

26. Xiaozhong F, Zero L, Guangling R et al (2012) Improvement of the synthesis process of benoxate. J Guiyang Med Coll 37(04):440-1 + 443

27. Teng SB, Qian J (2009) Progress in the synthesis of benoxylates. Shandong Chem Ind 38(08):23-25

28. Feng ZIL, Jiayuan X, Zhu ZB et al (2020) Synthesis of ginkgolide B structural modifiers and their effects on 6-OHDA-induced SH-SY5Y cell viability. Chin J Med Chem 30(04):199-203

29. Yong-Ming L, Jian P, Zhang, Wen-Na et al (2015) Synthesis, in silico and in vivo blood brain barrier permeability of ginkgolide $B$ cinnamate. Fitoterapia 106:110-114

30. Wu ZY, Pan J, Yuan Y, Hui A, Yang Y, Zhou A et al (2012) Brain-targeting research of 10-0-nicotinate ginkgolide B: a new prodrug of ginkgolide B. Med Chem Res 21(12):4028-4036

31. Sladowski D, Steer SJ, Clothier RH, Balls M (1993) An improved MTT assay. J Immunol Methods 157(1-2):203-207

\section{Figures}




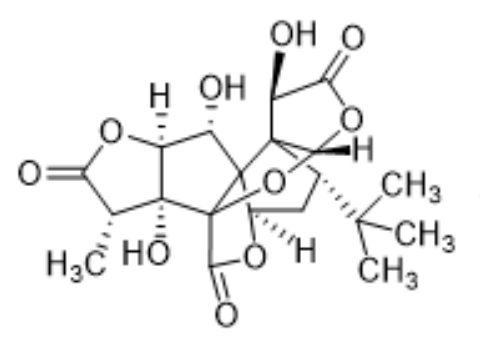

Ginkgolide B

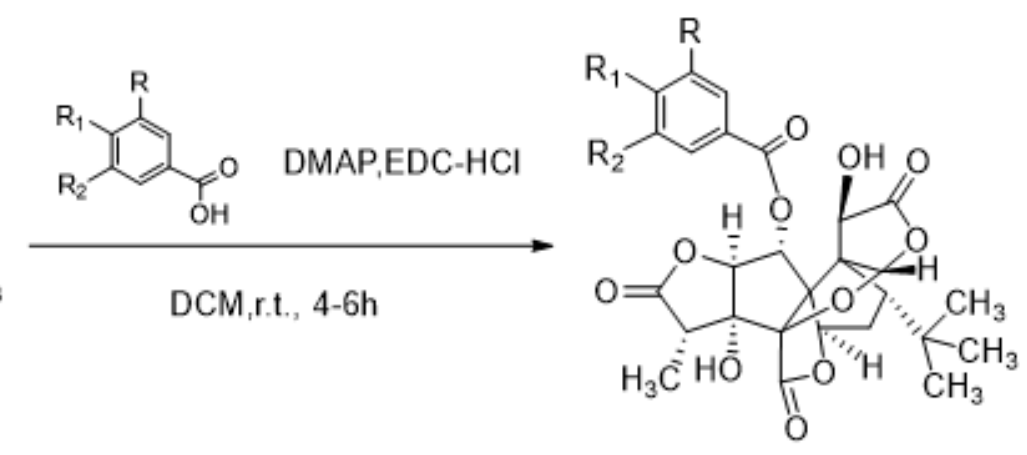

$\begin{array}{llcc} & \mathrm{R} & \mathrm{R}_{1} & \mathrm{R}_{2} \\ 1 & \mathrm{H} & \mathrm{Cl} & \mathrm{H} \\ 3 & \mathrm{H} & \mathrm{F} & \mathrm{H} \\ 5 & \mathrm{H} & \mathrm{OMe} & \mathrm{H} \\ 7 & \mathrm{H} & \mathrm{NO}_{2} & \mathrm{H} \\ 9 & \mathrm{OMe} & \mathrm{H} & \mathrm{H} \\ 11 & \mathrm{NO}_{2} & \mathrm{H} & \mathrm{NO}_{2}\end{array}$

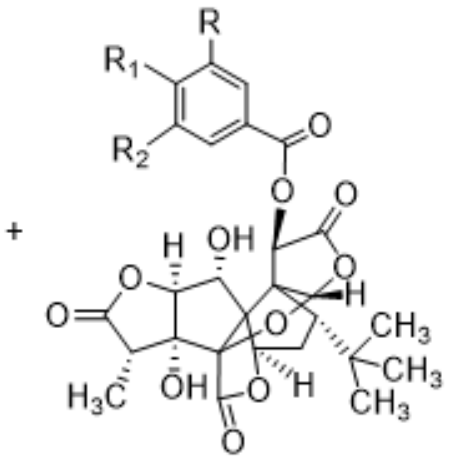

$\begin{array}{llcl} & \mathrm{R} & \mathrm{R}_{1} & \mathrm{R}_{2} \\ \text { 4-Chlorobenzoic acid } & \mathrm{H} & \mathrm{Cl} & \mathrm{H} \\ \text { 4-Fluorobenzoic acid } & \mathrm{H} & \mathrm{F} & \mathrm{H} \\ \text { 4-Methoxybenzoic Acid } & \mathrm{H} & \mathrm{OMe} & \mathrm{H} \\ \text { 4-nitrobenzoic acid } & \mathrm{H} & \mathrm{NO}_{2} & \mathrm{H} \\ \text { 3-Methoxybenzoic Acid } & \mathrm{OMe}^{\mathrm{H}} & \mathrm{H} \\ \text { 3,5-Dinitrobenzoic acid } & \mathrm{NO}_{2} & \mathrm{H} & \mathrm{NO}_{2}\end{array}$

$\begin{array}{cccc} & \mathrm{R} & \mathrm{R}_{1} & \mathrm{R}_{2} \\ 2 & \mathrm{H} & \mathrm{Cl} & \mathrm{H} \\ 4 & \mathrm{H} & \mathrm{F} & \mathrm{H} \\ 6 & \mathrm{H} & \mathrm{OMe} & \mathrm{H} \\ 8 & \mathrm{H} & \mathrm{NO}_{2} & \mathrm{H} \\ 10 & \mathrm{OMe} & \mathrm{H} & \mathrm{H}\end{array}$

\section{Figure 1}

Synthesis route of Ginkgolide B derivatives
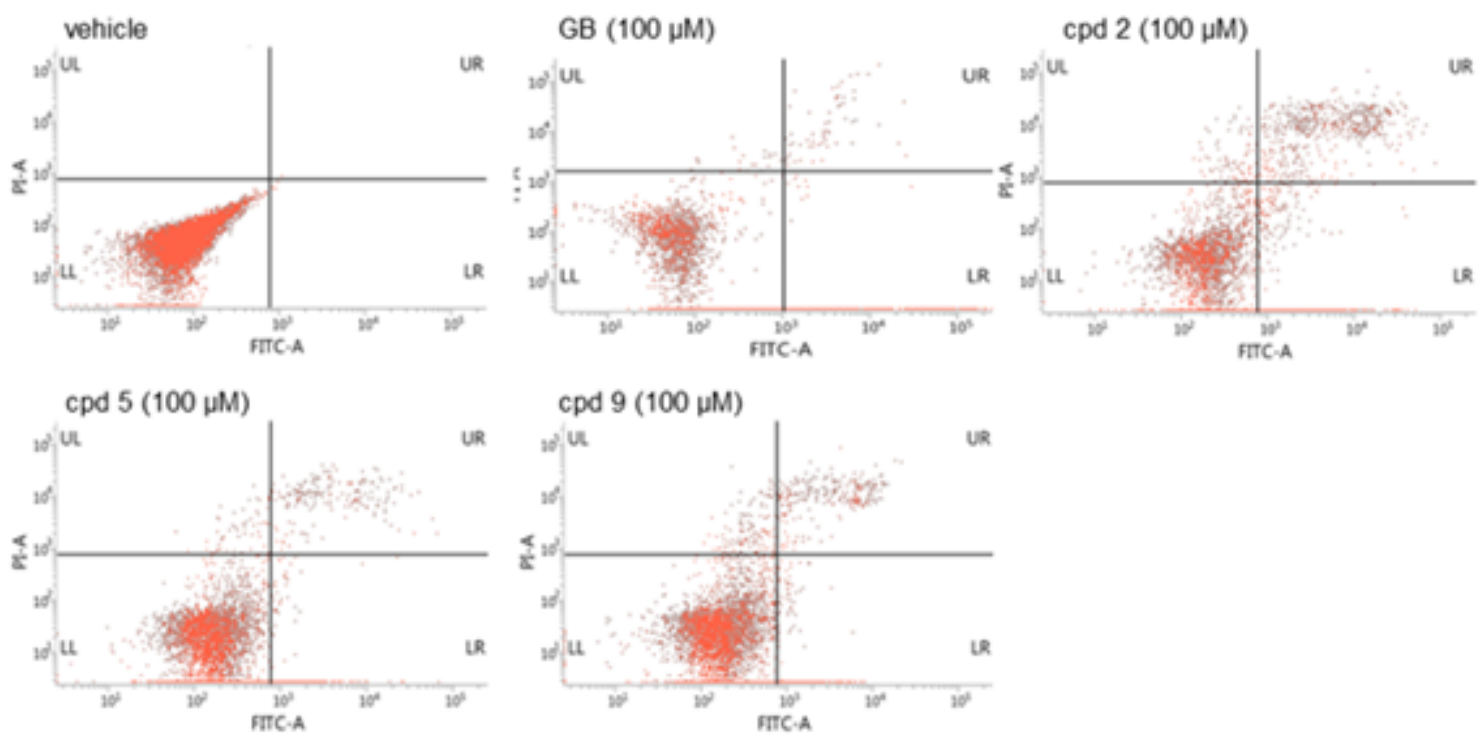

\section{Figure 2}

Annexin V/PI double staining assay to detect the effect of Ginkgolide B and its derivatives on the apoptosis of human SKOV3 cells. 